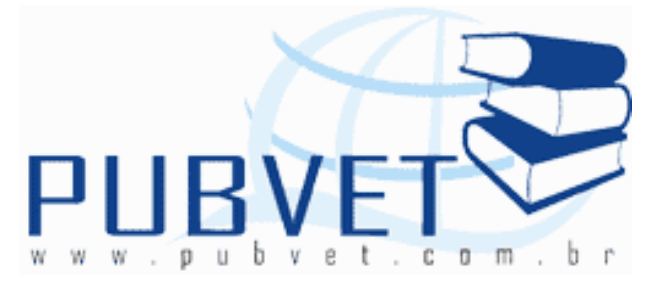

PUBVET, Publicações em Medicina Veterinária e Zootecnia.

\title{
Uso inadequado de Abamectina em bezerros Girolandos: Relato de caso
}

Rafael Lage Pereira Guerra ${ }^{1}$, Gabriel Domingos Carvalho ${ }^{2}$, Luciana Navajas Rennó de Araújo $^{3}$, Marco Túlio Lopes Serrano ${ }^{3}$

${ }^{1}$ Médico Veterinário formado pela Faculdade de Ciências Biológicas e da Saúde (FACISA/UNIVIÇOSA), Viçosa-MG.

${ }^{2}$ Professor do curso de Medicina Veterinária do Instituto Federal Norte de Minas Gerais - IFNMG Campus Salinas, Salinas-MG.

${ }^{3}$ Professor(a) do curso de Med. Veterinária da FACISA/UNIVIÇOSA, ViçosaMG.

Autor para correspondência: Gabriel Domingos Carvalho. Endereço: Fazenda Varginha, Km 02, Rod. Salinas/Taiobeiras. 39.560-000. Salinas, MG. Tel./Fax. +55 383841 7000. E-mail: gabriel.carvalho@ifnmg.edu.br

\section{Resumo}

Abamectina é um parasiticida injetável de amplo espectro cuja administração em pequeno volume controla eficazmente uma ampla variedade de parasitas que comprometem a saúde e a produtividade dos bovinos. Este trabalho tem como objetivo relatar 14 casos de intoxicação por abamectina em bezerros da raça girolanda, com idade inferior a três meses de idade, em uma propriedade leiteira do município de Jequetibá, Minas Gerais, Brasil. Os animais acometidos apresentaram apatia, anorexia e 
GUERRA, R.L.P. et al. Uso inadequado de Abamectina em bezerros Girolandos: Relato de caso. PUBVeT, Londrina, V. 5, N. 29, Ed. 176, Art. 1185, 2011.

ataxia e foram tratados com fluidoterapia. Dos 14 casos de intoxicação, 11 culminaram com o óbito dos animais.

Palavras-chave: abamectina, bovinos, intoxicação.

\title{
Inappropriate use of Abamectin in Girolanda calves: Case report
}

\begin{abstract}
Abamectin is an injectable parasiticide of wide spectrum that in a small volume administration controls effectively against a variety of parasites that endanger the health and productivity of cattle. This paper aims to report 14 cases of abamectin intoxication in girolanda calves, age less than three months old, on a dairy property in the city of Jequetibá state of Minas Gerais, Brazil. The affected animals presented apathy, anorexia and ataxia, and were treated with fluid therapy. From the 14 cases of intoxication, 11 resulted in death of the animals.
\end{abstract}

Keywords: abamectin, bovine, intoxication.

\section{INTRODUÇÃO}

As perdas econômicas causadas pelas parasitoses nos animais de produção são altas quando se considera a redução no ganho de peso e na produtividade, além do aumento na susceptibilidade a doenças. Desse modo, busca-se o controle das parasitoses a níveis aceitáveis, que não se alterem a produtividade (BALDANI et al., 1999).

A abamectina é um parasiticida injetável de amplo espectro cuja administração de pequeno volume controla eficazmente uma ampla variedade de parasitas que comprometem a saúde e a produtividade dos bovinos. É indicado para o tratamento e controle das parasitoses causadas por nematódeos gastrintestinais e pulmonares, bernes, piolhos, ácaros da sarna e como auxiliar no controle do carrapato bovino; auxiliando ainda, na prevenção do aparecimento de miíases em feridas decorrentes da castração ou descorna (PFIZER, 2009). 
GUERRA, R.L.P. et al. Uso inadequado de Abamectina em bezerros Girolandos: Relato de caso. PUBVeT, Londrina, V. 5, N. 29, Ed. 176, Art. 1185, 2011.

As avermectinas são descritas como possuírem uma ampla margem de segurança (LANKAS e GORDON, 1989; COURTNEY e ROBERSON, 1995; AYRES e ALMEIDA, 2002) porém, vários casos de intoxicação em bovinos tem sido relatados. O fármaco mais bem caracterizado do grupo das avermectinas é a ivermectina e pode induzir neurotoxicidade e alteração fetal em bovinos (RADOSTITS et al., 2002). A abamectina não é recomendada para bezerros com menos de quatro meses, dado o desenvolvimento incompleto da barreira hematoencefálica (PULLIAM e PRESTON, 1989; COURTNEY e ROBERSON, 1995; AYRES e ALMEIDA, 2002).

No Brasil o uso equivocado de abamectina em bezerros jovens têm resultado no aparecimento de casos de intoxicação iatrogênica, seguidos ou não de morte (SEIXAS et al., 2006). Para tanto se faz necessário estudos a respeito do assunto para melhor compreensão dos casos ocorridos.

Este trabalho tem como objetivo relatar 14 casos de intoxicação por abamectina em bezerros da raça girolanda, com idade inferior a três meses de idade, em uma propriedade leiteira do município de Jequetibá, Minas Gerais, Brasil.

\section{REVISÃO DE LITERATURA}

\subsection{As Avermectinas}

As avermectinas representam um grande progresso na terapia antiparasitária desde 1981, quando a ivermectina foi introduzida na medicina veterinária, mostrando-se eficiente contra um largo espectro de endo e ecto parasitas (TAYLOR, 2001; AYRES e ALMEIDA, 2002).

Etmologicamente, avermectina expressa, a (ausência) + verm (verme) + ect (externo) + in (quimioterápico), agrupando ivermectina, abamectina, doramectina, eprinomectina e selamectina. Sua síntese foi extraída da fermentação do fungo Streptomyces avermitilis, isoladas no solo do Japão, e posteriormente do território italiano (BURG et al., 1979).

A ivermectina foi à primeira entre as Lactonas Macrocíclicas (LM) a ser comercializada (CHABALA et al., 1980; EGERTON et al., 1980) e graças a 
GUERRA, R.L.P. et al. Uso inadequado de Abamectina em bezerros Girolandos: Relato de caso. PUBVET, Londrina, V. 5, N. 29, Ed. 176, Art. 1185, 2011.

seu amplo espectro de ação, eficácia, segurança clínica e novo modo de ação, tornou-se o tratamento de escolha para bovinos, ovinos, caprinos, suínos e eqüinos (CAMPBELL et al., 1983; CAMPBELL e BENZ, 1984).

As avermectinas são muito solúveis em gorduras corporais e essa característica é a razão para sua eficácia prolongada. Por outro lado, a longa retenção dos resíduos dessa droga, em produtos de origem animal, é problemático devido ao seu longo tempo de excreção pelo organismo e a conseqüente contaminação de alimentos, incluindo o leite além de causar intoxicação em animais muito jovens (CAMPBELL, 1989).

A toxicidade das avermectinas é, em parte, dependente na atividade da Glicoproteina-P. Esta se encontra localizada em alguns tecidos, inclusive na barreira hematoencefálica, canais hepatobiliares e placenta, agindo como uma proteína de efluxo, levando do interior para fora das células certas drogas. A importância da relação entre a toxicidade das avermectinas, com a Glicoproteina- P limita-se ao fato de que ela controla a entrada de avermectinas em tecidos potencialmente sensíveis. No SNC, é encontrada dentro dos capilares endoteliais formando a barreira hematoencefálica. Assim, a presença desta proteína, em capilares endoteliais encefálicas, afeta na suscetibilidade para os efeitos neurológicos agudos causados pelas avermectinas (RODRIGUES, 2007).

\subsection{Mecanismo de Ação e Farmacocinética das Avermectinas}

O mecanismo de ação das avermectinas ainda é discutido, supõe-se que o fármaco penetra nos parasitos gastrintestinais através da absorção transmuscular e dos hematófagos por via oral. As lactonas potencializam a ação inibidora neuronal no cordão nervoso ventral dos parasitos que é medida pelo ácido gama-aminobutírico (GABA) estes medicamentos agem sobre os canais de cloro, estimulam a liberação pré sináptica deste neurotransmissor, pelo aumento de sua ligação aos receptores póssinápticos, com isso aumenta a condução intracelular do neurotransmissor, 
GUERRA, R.L.P. et al. Uso inadequado de Abamectina em bezerros Girolandos: Relato de caso. PUBVeT, Londrina, V. 5, N. 29, Ed. 176, Art. 1185, 2011.

alterando a membrana do neurônio, resultando em uma paralisia motora do tipo flácida e eliminação do parasito (SPINOSA et al., 2002).

As avermectinas são insolúveis em água, mas se dissolvem bem em vários solventes orgânicos, como clorofórmio, acetona. A baixa hidrossolubilidade e elevada lipossolubilidade favorece a sua disposição no local de aplicação por via subcutânea ou pour on, o que prolonga o tempo de persistência do medicamento no organismo do animal. Este composto também é absorvido pelo sistema gastrintestinal, quando administrado por via oral, subcutânea e pour on, as concentrações elevadas são observadas no pulmão e na pele, a eliminação do fármaco é através das fezes e urina (SPINOSA et al., 2006).

\subsection{Abamectina}

A abamectina, também denominada de avermectina $B_{1 a}$, é um produto natural do processo de fermentação, servindo de partida para a produção de um análogo semi-sintético, 22,23 - dihidroavermecti $B_{1}$, ou ivermectina, cuja estrutura pode ser considerada como um híbrido entre os componentes $B_{1}$ e $B_{2}$. As soluções comerciais prontas para uso contém $1 \%$ de abamectina (componente $\mathrm{B}_{1 \mathrm{~A}}: \mathrm{C}_{48} \mathrm{H}_{72} \mathrm{O}_{14}$; componente $\mathrm{B}_{1 \mathrm{~B}}: \mathrm{C}_{47} \mathrm{H}_{70} \mathrm{O}_{14}$ ). É formulado para proporcionar a dosagem recomendada de $200 \mu \mathrm{g} / \mathrm{kg}$ de peso corpóreo, quando administrado pela via subcutânea, na proporção de um $\mathrm{mL} / 50 \mathrm{~kg}$ de peso corpóreo. A dosagem indicada é de $1 \mathrm{~mL}$ de Abamectina $1 \%$ para cada $50 \mathrm{~kg}$ de peso corpóreo (equivalente a $200 \mathrm{mg}$ de abamectina por $\mathrm{kg}$ de peso). O produto não deve ser utilizado em bezerros com menos de quatro meses de idade, ou com menos de $100 \mathrm{~kg}$ de peso (PFIZER, 2009).

\subsection{Toxicidade da Abamectina}

Experimentalmente, intoxicações aguda, subaguda e crônica, realizados com a abamectina em ratos, coelhos, cães e macacos demonstraram resultados similares aos da ivermectina, exceto pela maior toxidez desse fármaco. Em geral, as principais manifestações clínicas em 
GUERRA, R.L.P. et al. Uso inadequado de Abamectina em bezerros Girolandos: Relato de caso. PUBVET, Londrina, V. 5, N. 29, Ed. 176, Art. 1185, 2011.

bovinos são midríase, vômitos, tremores, ataxia, depressão, convulsões, coma e morte, com algumas variações entre as espécies (LANKAS e GORDON, 1989).

Na Austrália, Seaman et al. (1987) relataram casos de reações nervosas em um rebanho de bovinos da raça Murray Grey, após a administração de abamectina. Devido a ocorrência de mortes de animais com sintomatologia nervosa logo após a administração de doses terapêuticas de abamectina, os autores montaram um experimento e demonstraram altas concentrações do princípio ativo no cérebro dos animais, justificando os sinais clínicos apresentados.

Apesar das avermectinas apresentarem pouca distribuição no sistema nervoso central de mamíferos, doses altas podem desencadear efeitos tóxicos agudos com manifestações nervosas. Isso tem sido relatado como efeito no ácido gama-aminobutírico (GABA) nos cérebros de espécies mamíferas. Em bovinos, a abamectina provocou sinais de intoxicação administrada por via subcutânea na dose de $1,0 \mu \mathrm{g} / \mathrm{kg}$. Nos níveis de 2,0$8,0 \mu \mathrm{g} / \mathrm{kg}$ e maiores, provocou sinais mais sérios como ataxia, decúbito, coma e morte. Os produtos formulados com abamectina alertam contra o uso em bezerros abaixo de quatro meses de idade (LANKAS e GORDON, 1989; SHOOP e SOLL, 2002).

\section{RELATO DE CASO}

Este trabalho descreve 14 casos de intoxicação por abamectina em bezerros da raça girolanda, com idade inferior a três meses de idade, ocorridos em uma propriedade leiteira do município de Jequetibá, região central metelurgica do estado de Minas Gerais, Brasil. Esta propriedade possui 800 hectares de área, com uma produção média de aproximadamente 2.000 litros de leite por dia, possuindo dois currais. O curral do gado girolando possui 65 vacas na ordenha, produzindo 1.100 litros/dia, 27 bezerros mamando no bezerreiro e seis funcionários. A fazenda possui assistência médico veterinária com duas visitas ao mês. Todos os 
GUERRA, R.L.P. et al. Uso inadequado de Abamectina em bezerros Girolandos: Relato de caso. PUBVET, Londrina, V. 5, N. 29, Ed. 176, Art. 1185, 2011.

animais da propriedade são submetidos a um programa sanitário, que inclui um calendário de vacinação com controle, sem notificação, de doenças como rinotraqueíte infecciosa bovina (IBR), diarréia viral bovina (BVD), brucelose, tuberculose, febre aftosa, leptospirose, raiva e clostridioses.

Os bezerros eram criados em casinhas individuais, a 50 metros do curral e recebiam leite duas vezes ao dia e tinham ração e feno a vontade, não tendo contato com outros animais. Foi prescrito pelo médico veterinário responsável a aplicação mensal de ivermectina, por via subcutânea, na dosagem de $1 \mathrm{~mL}$ para os bezerros até 30 dias e $2 \mathrm{~mL}$ para os animais acima de 30 dias, porém, o funcionário responsável pela ordenha e pelo trato fez a administração de abamectina nessa mesma dosagem.

Os animais manifestavam sinais clínicos nas primeiras $24 \mathrm{~h}$ após a aplicação do medicamento. Os sinais clínicos observados foram inapetência, apatia, ataxia e morte dos indivíduos. Do total de animais acometidos, 11 progrediram para a morte.

$\mathrm{Na}$ Tabela 1 pode-se observar a identificação dos animais, doses aplicadas e desfecho dos casos.

Tabela 1. Identificação dos animais, doses aplicadas e desfecho do caso.

\begin{tabular}{lccc}
\hline Animal & $\begin{array}{c}\text { Peso ao nascer } \\
(\mathbf{K g})\end{array}$ & $\begin{array}{c}\text { Idade ao óbito } \\
\text { (dias) }\end{array}$ & Desfecho do caso \\
\hline Ma0101 & 29 & 43 & Óbito \\
814 & 32 & 50 & Óbito \\
971 & 35 & 56 & Óbito \\
Ma0015 & 27 & 75 & Óbito \\
Y527 & 33 & 37 & Óbito \\
Y941 & 28 & 40 & Óbito \\
Y840 & 33 & 62 & Óbito \\
Ma 052 & 36 & 69 & Óbito \\
Ma 050 & 31 & 35 & Óbito \\
Y 872 & 31 & 43 & Óbito \\
Y 077 & 29 & 45 & Óbito \\
812 & 26 & - & Sobrevivente \\
815 & 33 & - & Sobrevivente \\
818 & 35 & - & Sobrevivente \\
\hline
\end{tabular}


GUERRA, R.L.P. et al. Uso inadequado de Abamectina em bezerros Girolandos: Relato de caso. PUBVeT, Londrina, V. 5, N. 29, Ed. 176, Art. 1185, 2011.

\section{RESULTADOS E DISCUSSÃO}

Inicialmente, suspeitou-se, pelos funcionários da fazenda, de tratar-se de tristeza parasitária bovina (TPB). Essa hipótese foi descartada pela falta de sintomatologia compatível com a mesma, como febre, mucosasa hiperêmicas, hemoglobinúria, e pelo surgimento de outros sinais clínicos após a administração do medicamento.

Os 11 animais que morreram, vieram a óbito em até cinco dias após a administração do medicamento, e possuíam idade inferior a três meses. $O$ uso de abamectina não é recomendado para bezerros com menos de quatro meses de idade (PULLIAM e PRESTON, 1989; COURTNEY e ROBERSON, 1995; AYRES e ALMEIDA, 2002).

Quando se identificou que o problema era a medicação incorreta, os animais acometidos começaram a ser tratados para o quadro sintomático de intoxicação. Realizou-se fluidoterapia parenteral com suplementação vitamínica e energética e outros cuidados de enfermagem (fornecimento de água, leite por sonda e mudança de decúbito).

Conforme demonstrado por Seixas et al. (2006), no Brasil o uso equivocado de abamectina em bezerros jovens têm resultado em casos de intoxicação seguidos ou não de morte, como o relatado nesse trabalho.

Três bezerros sobreviveram, porém o seu ganho de peso ficou prejudicado, quando comparado com o peso de outros bezerros sadios presentes na mesma propriedade, ficou abaixo da média que é de $150 \mathrm{Kg}$ para a idade aproximada de 180 dias (Tabela 2).

Tabela 2. Condição de crescimento dos animais sobreviventes. Animal Idade em dias Peso em kg

812

815

818

Média da Propriedade
184

173

192

180
109

103

115

150 
GUERRA, R.L.P. et al. Uso inadequado de Abamectina em bezerros Girolandos: Relato de caso. PUBVET, Londrina, V. 5, N. 29, Ed. 176, Art. 1185, 2011.

\section{CONCLUSÕES}

O uso de abamectina deve ser realizado sob a prescrição e orientação de um profissional médico veterinário, e esta deve ser seguida corretamente. O uso inadequado pode acarretar em prejuízos como a intoxicação e morte dos animais com idade inferior a dezesseis semanas de vida.

Deve-se considerar também que os sinais clínicos manifestados são inespecíficos e sem uma causa aparente, sendo necessária uma investigação aprofundada para o esclarecimento dos casos.

\section{REFERÊNCIAS}

AYRES, M.C.C.; ALMEIDA, M.A.O. Agentes antinematódeos, Agentes antiparasitários. In: SPINOSA, H.S.; GÓRNIAK, S.L. e Bernadi, M.M. (Eds.). Farmacologia Aplicada à Medicina Veterinária. 3ed. Rio de Janeiro: Guanabara Koogan, 2002. p.476-488.

BALDANI, L.A.; SOUSA, R.V.; MIGUEL, A.G. Farmacologia dos principais antiparasitários de uso na medicina veterinária. Boletim informativo da UFLA, n.42. Lavras: UFLA, 1999. 39p.

CAMPBELL, W.C. Ivermectin and abamectin. New York: Springer-Verlag. 1989. 363 p.

CAMPBELL, W.C.; BENZ, G.W. Ivermectin: a review of efficacy and safety. Journal of Veterinary Pharmacology and Therapeutics, v.7, p.1-16, 1984.

CAMPBELL, W.C.; FISHER, M.H.; STAPLEY, E.O.; ALBERS-SCHONBERG, G.; JACOBS, T.A. Ivermectin: a potent new antiparasitic agent. Science, v.221, p.823-828, 1983.

CHABALA, J.C.; MROZIK, H.; TOLMAN, R.L.; ESKOLA, P.; LUSI, A.; PETERSON, L.H,; WOODS, M.F.; FISHER, M.H. Ivermectin, a new broad-spectrum antiparasitic agent. Journal of Medicinal Chemistry, v.23, p.1134-1136, 1980.

COURTNEY, C.H.; ROBERSON, E.L. Chemotherapy of parasitic disease. Antinematodal drugs. In: ADAMS, H.R. (ed.) Veterinary Pharmacology and Therapeutics. 7ed. Ames: Iowa State University Press, 1995. p.916-922.

EGERTON, J.R.; BIRNBAUM, J.; BLAIR, L.S.; CHABALA, J.C.; CONROY, J.; FISHER, M.H.; MROZIK, H.; OSTLIND, D.A.; WILKINS, C.A.; CAMPBELL, W.C. 22,23-Dihydroavermectin B1, a new broad-spectrum antiparasitic agent. British Veterinary Journal, v.136, p.8897, 1980.

FISHER, M.H.; MROZIK, H. Chemistry. In: CAMPBELL, W.C. (ed.) Ivermectin and abamectin. New York: Springer-Verlag, 1989. p.1-23.

LANKAS, G.R.; GORDON, L.R. Toxicology. In: CAMPBELL, W.C. (ed.) Ivermectin and Abamectin. New York: Springer-Verlag, p.89-112.

PFIZER - Saúde Animal. Bula: Abamectina 1\% Pfizer. Disponível em: http://www.pfizersaudeanimal.com.br/. Acesso em: 2 de outubro de 2009.

PULLIAM, J.D.; PRESTON, J.M. 1989. Safety of ivermectin in target animals. In: Campbell W.C. (ed.) Ivermectin and Abamectin. New York: Springer-Verlag, 1989. p.149161. 
RADOSTITS, O.M.; GAY, C.C.; BLOOD, D.C.; HINCHCLIFF, K.W. 2002. Doenças causadas por substâncias químicas inorgânicas e produtos químicos utilizados nas fazendas. In: Ibid. (ed.) Clínica Veterinária: um tratado de doenças dos bovinos, ovinos, suínos, caprinos e eqüinos. 9ed. Rio de Janeiro: Guanabara Koogan, 2002. p.1417-1471.

RODRIGUES, D.C. Avaliação da toxicidade de avermectinas em bovinos com idade inferior a trinta dias. 63f.: il.; $28 \mathrm{~cm}$. Dissertação (Mestrado) - Universidade Estadual Paulista, Faculdade de Ciências Agrárias e Veterinárias, Jaboticabal, 2007.

SEAMAN, J.T.; EAGLESON, J.S.; CARRIGAN, M.J.; WEBB, R.F. Avermectin B1 toxicity in a herd of Murray Grey cattle. Australian Veterinary Journal, v.64, n.9, p.284-285, 1987.

SEIXAS, J.N.; PEIXOTO, P.V.; ARMIÉN, A.G.; JABOUR, F.F.; BRITO, M.F. Aspectos clínicos e patogenéticos da intoxicação por abamectina em bezerros. Pesquisa Veterinária Brasileira, v.26, n.3. p.161-166, 2006.

SHOOP, W.L.; SOLL, M. Chemistry, pharmacology and safety of the macrocyclic lactones. In: VERCRUYSSE, J.; REW, R.S. (ed.s). Macrocyclic lactones in antiparasitic therapy. Wallingford: CAB International, 2002. p.1-29.

SPINOSA, H.S.; GORNIAK, S.L.; BERNARDI, M.M. Farmacologia Aplicada À Medicina Veterinária. 3ed. Rio de Janeiro: Guanabara Koogan, 2002. 752 p.

SPINOSA, H.S.; GORNIAK, S.L.; BERNARDI, M.M. Farmacologia Aplicada À Medicina Veterinária. 4ed. Rio de Janeiro: Guanabara Koogan, 2002. 897 p.

TAYLOR, M.A. Review: recent developments in ectoparasites. Veterinary Journal, v.161, p.253-268, 2001. 